\title{
Reviewing a patient with coeliac disease
}

\author{
James W Berrill specialist registrar in gastroenterology ${ }^{1}$, Haroon Ahmed specialty academic trainee \\ in general practice ${ }^{2}$, Sohail Butt general practitioner ${ }^{3}$, Gillian Swift consultant gastroenterologist ${ }^{1}$
}

${ }^{1}$ University Hospital Llandough, Cardiff CF64 2XX, UK; ${ }^{2}$ Foundry Town Clinic, Aberdare, UK; ${ }^{3}$ Studholme Medical Centre, Ashford, UK

This is part of a series of occasional articles on common problems in primary care. The $B M J$ welcomes contributions from GPs.

A 44 year old woman diagnosed with coeliac disease 10 years ago presents to her general practitioner with symptoms of bloating and diarrhoea that have developed over the past two months.

\section{What you should cover}

Coeliac disease is a common gastrointestinal disease, with international population studies reporting a prevalence of $0.5-1 \%$. Inflammation of the small bowel mucosa occurs as a result of an immunological response to dietary gluten. The mainstay of treatment is a gluten-free diet.

The most appropriate method of follow-up for patients with coeliac disease is still debated. Evidence suggests that regular follow-up improves compliance with a gluten-free diet. Survey data have shown that patients would prefer a model allowing regular follow-up with a dietitian, with specialist medical expertise available if needed. However, limited resources and the ability of patients to self manage means that many patients consult their general practitioner only when they have concerns. This consultation presents an opportunity to review their management.

In patients with a relapse of symptoms:

- Reconsider the diagnosis: is this definitely coeliac disease? Review the levels of antibodies to tissue transglutaminase and the histological findings.

- Ascertain whether symptoms improved after starting a gluten-free diet; most patients report improvement of symptoms in the first few weeks. Of those whose symptoms do not improve with the diet, only a very small minority have true refractory coeliac disease, with about $90 \%$ having an alternative underlying cause identified.

- Discuss the patient's diet. The commonest cause of persistent or recurring symptoms is ingestion of gluten. Ongoing exposure to gluten will usually result in raised levels of tissue transglutaminase antibodies. A gluten-free diet can be a major undertaking for patients, so explore and resolve any perceived barriers or difficulties (such as availability of gluten-free foods, impact on social life and "eating out," and social stigma). A review by a dietitian is essential as following a gluten-free diet will require education on which foods contain gluten and how to obtain gluten-free products. Highlight further sources of information and support, such as the association Coeliac UK.

- Acknowledge that some patients may not wish to follow a gluten-free diet or may wish to have phases of compliance depending on their symptoms. Explain the long term risks associated with this strategy and help patients to make an informed decision, providing support as needed. Explain that many food products do not contain gluten, such as fruit, egg, cheese, vegetables, meat, and fish.

- Identify if the patient has any "red flag" symptoms that require urgent investigation to exclude alternative gastrointestinal disease. Ask about vomiting, rectal bleeding, and weight loss. The diagnosis of coeliac disease does not exclude coincidental disease, and for any patients developing new alarm symptoms or iron deficiency anaemia consider referral for further investigation.

\section{What you should do \\ Clinical examination}

- Identify signs of nutritional deficiency (oral aphthous ulceration, angular stomatitis, koilonychia) and skin rashes (dermatitis herpetiformis).

- Record the patient's weight so that objective evidence of change in weight will be available.

\section{Blood tests}

- Arrange a full blood count, ferritin, vitamin $\mathrm{B}_{12}$, and folate. Half of patients with coeliac disease are anaemic at presentation, and although their anaemia will improve if 
they follow a gluten-free diet, specific deficiencies may need to be corrected to aid recovery.

- Arrange liver function tests. Transient hepatitis may be present at diagnosis and will resolve with introduction of a gluten-free diet; however, persistent abnormalities may be the result of associated autoimmune conditions such as primary biliary cirrhosis or autoimmune hepatitis.

- Check glucose and thyroid function levels because of the association of coeliac disease with diabetes and autoimmune thyroid disease.

- Check tissue transglutaminase antibody levels if ongoing gluten ingestion is suspected.

\section{Management}

For patients who have difficulty adhering to a gluten-free diet, offer referral to a dietitian. The types of food that can be prescribed have been reviewed recently; for information see Coeliac UK's revised prescribing guide ("Useful resources" box). In some regions, supply of gluten-free products is led by community pharmacies. The table $\Downarrow$ shows examples of monthly prescriptions for different patient groups, according to the number of "units" of gluten-free food products they need (units are allocated to each prescribable food on the basis of its carbohydrate and energy content and its cost).

Pancreatic insufficiency can be associated with coeliac disease and presents with diarrhoea or steatorrhoea. To diagnose pancreatic insufficiency send a stool sample for measurement of faecal elastase; if the level is low, consider a trial of pancreatin granules (such as Creon; Abbott Healthcare) - the dose can be adjusted to control symptoms, and a dietitian's advice is useful.

Although small bowel lymphoma is more common in people with coeliac disease, the annual incidence in the general population is low (0.5-1 per million), resulting in a very small increase in absolute risk for patients with coeliac disease. The increased risk reduces to a normal level after a gluten-free diet has been followed for three years. However, unexplained weight loss, abdominal pain, a palpable mass, and hypoalbuminaemia should alert you to the possibility of lymphoma and warrants referral for investigation (tissue transglutaminase antibody levels may be normal in this scenario).
If the cause of symptoms is still not clear, referral for gastroenterology opinion may be needed to exclude other associated conditions such as lactose intolerance, microscopic colitis, small bowel bacterial overgrowth, or irritable bowel syndrome. Seek specialist opinion if you suspect refractory coeliac disease.

\section{Longer term aspects of management}

The risk of developing osteoporosis is substantial, with $40 \%$ of patients having decreased bone mineral density at the time of diagnosis. However, in most cases the density will improve with a gluten-free diet. Current guidance suggests measurement of bone mineral density in all patients at diagnosis, with the subsequent frequency of assessment dictated by the baseline result together with patients' other risk factors for developing osteoporosis. Discuss relevant lifestyle measures, including regular exercise, reducing alcohol intake, and smoking cessation, and prescribe calcium supplements if necessary to ensure a daily intake of $1500 \mathrm{mg}$.

About $30 \%$ of patients with coeliac disease have hyposplenism. As a result of this increased prevalence and the consequent susceptibility to infection, England's Department of Health recommends offering pneumococcal vaccination to all patients, and some experts also suggest offering vaccines against haemophilus and influenza.

Discuss the risk of family members developing coeliac disease. First degree relatives have a 1 in 10 chance of being affected and should be assessed if symptoms develop.

Competing interests: All authors have completed the ICMJE uniform disclosure form at www.icmje.org/coi_disclosure.pdf (available on request from the corresponding author) and declare: no support from any organisation for the submitted work; SB performs consultancy as a primary care adviser to Juvela (a gluten-free food manufacturer); SB and GS are members of the health advisory committee to Coeliac UK. Provenance and peer review: Not commissioned; externally peer reviewed.

Accepted: 15 November 2011

Cite this as: BMJ 2012;344:d8152

(c) BMJ Publishing Group Ltd 2012 


\section{Useful resources}

- Primary Care Society of Gastroenterology. The management of adults with coeliac disease in primary care. 2006. www.pcsg.org.uk/ downloads/pcsg-publications.

- British Society of Gastroenterology. Management of adults with coeliac disease. 2010. www.bsg.org.uk/images/stories/clinical/bsg_ coeliac_10.pdf

- National Institute for Health and Clinical Excellence. Recognition and assessment of coeliac disease. (Clinical guideline 86.$) 2009$. http://guidance.nice.org.uk/CG86.

- Coeliac UK (www.coeliac.org.uk/). Charity providing help and support to people with coeliac disease.

- Coeliac UK. Gluten-free foods: a revised prescribing guide 2011. www.coeliac.org.uk/sites/files/coeliac/prescribing_guidelines_2.pdf.

\section{Table}

\begin{tabular}{|c|c|c|}
\hline Age group (years) & Recommended number of units ${ }^{*}$ & Example of monthly prescription \\
\hline $1-3$ & 10 & $6 \times 400 \mathrm{~g}$ bread, $2 \times 500 \mathrm{~g}$ pasta \\
\hline $4-6$ & 11 & $6 \times 400 \mathrm{~g}$ bread, $2 \times 500 \mathrm{~g}$ pasta, $1 \times(2 \times 110-180 \mathrm{~g}$ pizza bases $)$ \\
\hline $7-10$ & 13 & $8 \times 400 \mathrm{~g}$ bread, $2 \times 500 \mathrm{~g}$ pasta, $1 \times(2 \times 110-180 \mathrm{~g}$ pizza bases $)$ \\
\hline $11-14$ & 15 & $8 \times 400 \mathrm{~g}$ bread, $3 \times 500 \mathrm{~g}$ pasta, $1 \times(2 \times 110-180 \mathrm{~g}$ pizza bases $)$ \\
\hline $15-18$ & 18 & $8 \times 400 \mathrm{~g}$ bread, $4 \times 500 \mathrm{~g}$ pasta, $2 \times(2 \times 110-180 \mathrm{~g}$ pizza bases $)$ \\
\hline \multicolumn{3}{|l|}{ Men } \\
\hline $19-59$ & 18 & $\begin{array}{l}8 \times 400 \mathrm{~g} \text { bread, } 4 \times 500 \mathrm{~g} \text { pasta, } 1 \times(2 \times 110-180 \mathrm{~g} \text { pizza bases }), 1 \times 200 \mathrm{~g} \\
\text { crackers or crispbreads }\end{array}$ \\
\hline $60-74$ & 16 & $8 \times 400 \mathrm{~g}$ bread, $2 \times 500 \mathrm{~g}$ pasta, $2 \times 200 \mathrm{~g}$ crackers or crispbreads \\
\hline$\geq 75$ & 14 & $8 \times 400 \mathrm{~g}$ bread, $2 \times 500 \mathrm{~g}$ pasta, $2 \times 200 \mathrm{~g}$ crackers or crispbreads \\
\hline \multicolumn{3}{|l|}{ Women } \\
\hline $19-74$ & 14 & $8 \times 400 \mathrm{~g}$ bread, $2 \times 500 \mathrm{~g}$ pasta, $2 \times 200 \mathrm{~g}$ crackers/crispbreads \\
\hline$\geq 75$ & 12 & $6 \times 400 \mathrm{~g}$ bread, $2 \times 500 \mathrm{~g}$ pasta, $2 \times 200 \mathrm{~g}$ crackers/crispbreads \\
\hline
\end{tabular}

*For prescribing purposes, each prescribable gluten-free food is allocated a unit on the basis of its carbohydrate and energy content and its cost. Thus: $400 \mathrm{~g}$ bread $=1$ unit; $500 \mathrm{~g}$ pasta $=2$ units; two pizza bases $(110-180 \mathrm{~g}$ each $)=1$ unit; $200 \mathrm{~g}$ crackers or crispbreads $=1$ unit. For breast feeding, add an extra four units to the mother's requirement, and for third trimester of pregnancy add one unit. 